\title{
An unusual large erupting complex odontome in posterior maxilla: a case report
}

\begin{abstract}
Odontomas are the most common type of odontogenic tumor. The lesions are invariably asymptomatic. The lesions rarely grow to a large size and erupt into the oral cavity. Here we present a case of large complex odontome in the maxillary posterior most area which erupted into the oral cavity.
\end{abstract}

Keywords: odontome, complex odontome, benign odontogenic tumour
Volume 7 Issue I - 2017

\author{
Amit Mohan,' Sumit Grover² \\ 'Department of Oral and Maxillofacial Surgery, Manav Rachna \\ Dental College, India \\ ${ }^{2}$ Department of Orthodontics, Ashok Nursing Home, India
}

Correspondence: Amit Mohan, Department of Oral and Maxillofacial Surgery, Manav Rachna Dental College, Sector 46, Faridabad, Haryana, India, Tel 9| 97। I I 19707,

Email dr.mohanamit@gmail.com

Received: September 07, 2016 | Published: April 18, 2017

\section{Introduction}

Odontomas are the most common type of odontogenic tumor occurring within the jaws, commonly classified as-compound and complex odontome. The Compound odontomas are more common in the anterior portion of the jaws resembling tooth-like structure. The Complex odontomas are more common in the posterior part of the jaws and consist of a disorganized mass with no morphologic resemblance to a tooth. ${ }^{1}$ The lesions are invariably asymptomatic and are discovered on routine radiographic examinations; however, they may be associated with pathologic changes in adjacent teeth and structures such as-malformation, impaction, delayed eruption, displacement, cyst formation and resorption of adjacent teeth. ${ }^{2}$ In extremely rare instances odontome are larger than the tooth and may erupt into the oral cavity. ${ }^{3}$ In this manuscript we have reported and discussed one such unusual case of erupting large complex odontome in the maxillary posterior area.

\section{Case report}

A 25 year old female reported with the complaint of pain and swelling in left maxillary posterior region with feeling of a hard tooth-like structure since $1 \frac{1}{2}$ to 2 years. On examination, a large, well-circumscribed, non-capsulated, yellowish-white hard mass was noted in left maxillary posterior region (Figure 1). The mass was approximately $2.5 \mathrm{~cm}$ in size, was immobile and non-tender. On radiographic examination, a large well-defined radiopaque mass in left maxillary posterior region (Figure $2 \& 3$ ). The left maxillary $3^{\text {rd }}$ molar was located on the superior aspect of the mass. The clinical and radiological features were suggestive of complex odontome. Surgical removal of the lesion and impacted $3^{\text {rd }}$ molar was planned under local anaesthesia. The lesion was enucleated as a single mass (Figure 4). The impacted left maxillary $3^{\text {rd }}$ molar was also removed after enucleation of the lesion.

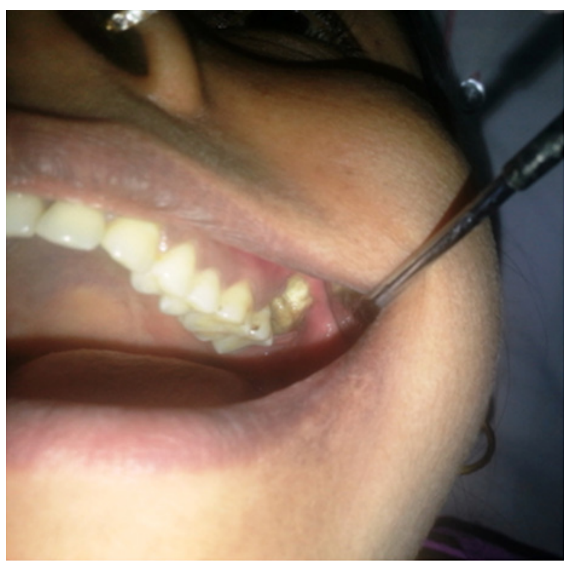

Figure I Clinical examination of the lesion.

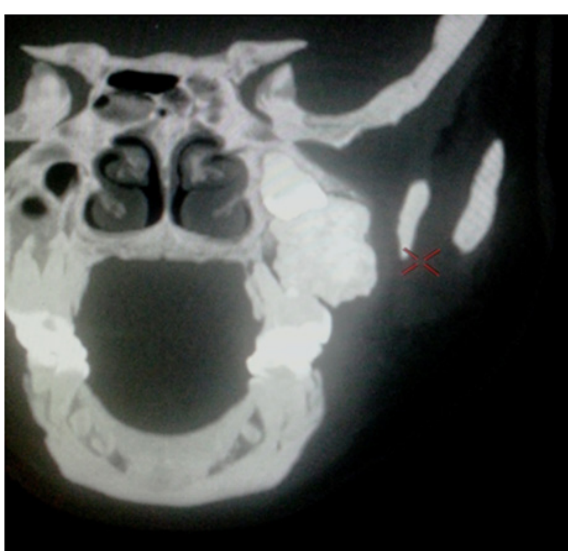

Figure 2 CT scan of the lesion. 


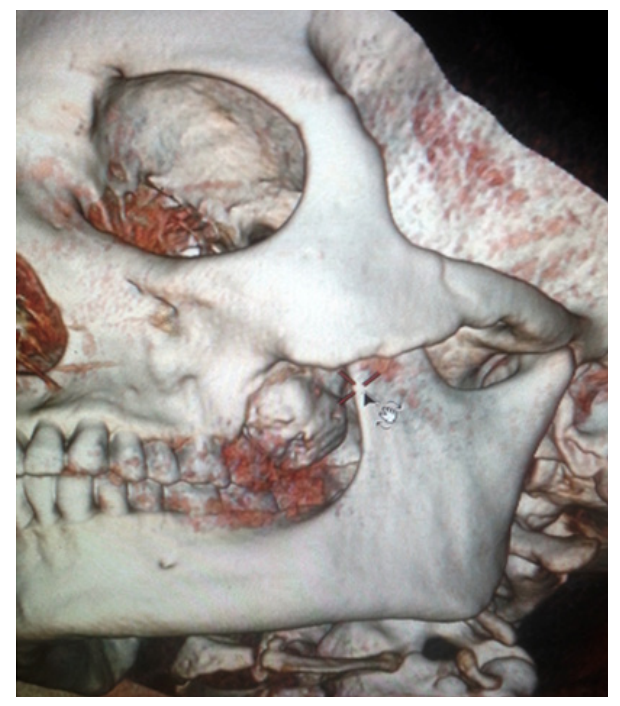

Figure 3 3D-CT scan of the lesion.

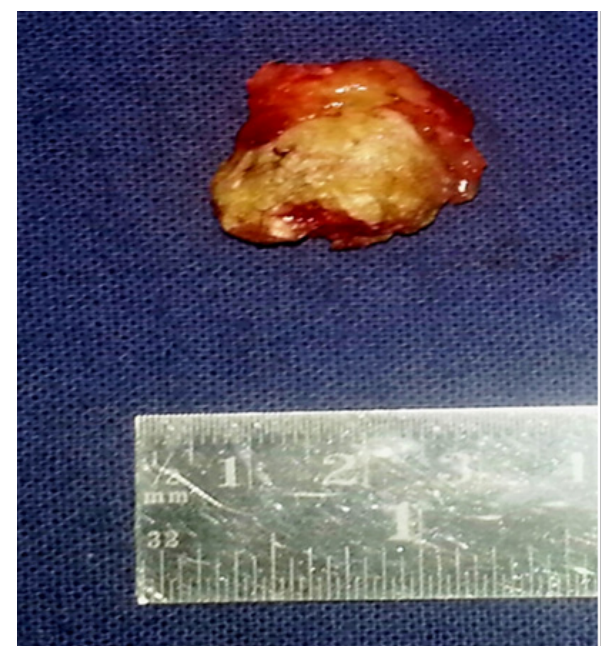

Figure 4 Gross specimen after removal.

\section{Discussion}

The term 'odontome' was coined by Paul Broca in 1867. The etiology of odontome remains unknown. Majority of the cases are discovered before the age of 30 years and there is slight predominance in females. ${ }^{4}$ They are usually asymptomatic but often associated with eruption disturbances, as seen in our case-a large complex odontome interfering with the eruption of maxillary $3^{\text {rd }}$ molar. Odontome and the possible disturbances to the adjacent teeth can be detected on plain radiograph. However, in our case, a CT scan was advised to confirm the relationship with adjacent teeth, pterygoid plates and the maxillary sinus. The differential diagnosis of complex odontoma include cementifying or ossifying fibroma, adenomatoid odontogenic tumor, calcifying odontogenic cysts, periapical cemental dysplasia and calcifying epithelial odontogenic tumor. Odontomas erupting into the oral cavity are rare. The possible mechanism could be the enlarging size, causing resorption of the surrounding bone. ${ }^{5}$ Another possible cause for eruption could be the eruptive forces of the apparently impacted maxillary $3 \mathrm{rd}$ molar teeth. The treatment of odontome, if required, is surgical removal. In our case the removal of impacted $3^{\text {rd }}$ molar was also done along with the surgical removal of odontome due to its unfavorable apically displaced position, anticipating its failure to erupt normally into the oral cavity.

\section{Acknowledgments}

None.

\section{Conflicts of interest}

The authors declare there is no conflict of interest.

\section{References}

1. Serra-Serra G, Berini-Aytés L, Gay-Escoda C. Erupted odontomas: A report of three cases and review of the literature. Med Oral Patol Oral Cir Bucal. 2009;14(6):E299-E303.

2. Budnick. Compound and complex odontomas. Oral Surg Oral Med Oral Pathol. 1976;42(4):501-506.

3. Kaneko M, Fukuda M, Sano T, et al. Microradiographic and microscopic investigation of a rare case of complex odontoma. Oral Surg Oral Med Oral Pathol Oral Radiol Endod. 1998;86(1):131-134.

4. Reichart AP, Philipsen HP. Odontogenic tumors and allied lesions. Quintessence, London, England, UK, 2004:1-338.

5. Vengal M, Arora H, Ghosh S, et al. Large erupting complex odontoma: a case report. J Can Dent Assoc. 2007;73(2):169-173. 